Résumés des conférences et travaux

\title{
Vladimir Vodoff
}

(9 mai 1935-14 janvier 2009)

\section{Pierre Gonneau}

\section{(2) OpenEdition \\ 1 Journals}

Édition électronique

URL : https://journals.openedition.org/ashp/954

DOI : 10.4000/ashp.954

ISSN : 1969-6310

Éditeur

Publications de l'École Pratique des Hautes Études

\section{Édition imprimée}

Date de publication : 2 février 2011

Pagination : xvii-xix

ISSN : 0766-0677

\section{Référence électronique}

Pierre Gonneau, «Vladimir Vodoff », Annuaire de l'École pratique des hautes études (EPHE), Section des sciences historiques et philologiques [En ligne], 141 | 2011, mis en ligne le 22 février 2011, consulté le 06 juillet 2021. URL : http://journals.openedition.org/ashp/954; DOI : https://doi.org/10.4000/ashp.954 


\section{VLADIMIR VODOFF \\ (9 MAI 1935-14 JANVIER 2009)}

$\mathrm{V}$

LADIMIR VODOFF incarnait, avec plusieurs autres membres de sa génération, le mariage de l'émigration russe avec l'Université française. Élevé dans une famille dont les racines remontaient à Viatka du côté maternel et à Odessa du côté paternel, il était aussi un parisien de naissance et devint, au fil de ses études, un représentant de l'école historique française dont il appréciait l'esprit et les méthodes. C'est ainsi qu'il fut élève de l'École nationale des chartes de 1954 à 1958. La thèse d'école qu'il y soutint, signalée au ministre, s'intitulait « Recherches sur les représentations scéniques de la crêche et les chants de la Nativité en Ukraine au XVIII ${ }^{\mathrm{e}}$ siècle et leur expansion ». Le jury associait le chartiste Robert Bossuat, et le slaviste André Vaillant, qui occupa le poste de directeur d'études à l'École pratique des hautes études pendant un bail exceptionnellement long (1932-1966). André Vaillant fut, avec André Mazon et Pierre Pascal, l'un des maîtres qui exercèrent une influence durable sur Vladimir Vodoff dont la carrière s'orienta bientôt vers l'enseignement. En effet, alors qu'à sa sortie de l'École des chartes, il avait opté pour le monde des bibliothèques, il se laissa rapidement attirer vers d'autres horizons. Reçu en 1959 à l'agrégation de russe, il débuta naturellement dans l'enseignement secondaire, à une époque où sa langue maternelle était en plein essor dans notre système éducatif. Toutefois, il ne s'éloigna pas longtemps de la Sorbonne. En effet, en 1968, il obtenait une charge de conférences à l'École pratique des hautes études, dans le cadre de la direction de Jacques Lépissier qui venait de remplacer André Vaillant. Fidèle à sa formation chartiste, Vladimir Vodoff intitulait sa conférence «Paléographie et diplomatique russes médiévales ». Après un début mouvementé, en une année où le Quartier Latin connut quelques soubresauts, la conférence prenait à peine sa vitesse de croisière quand un nouveau tournant se produisit. En effet, en très peu de temps Vladimir Vodoff devint chargé d'enseignement à l'université Lille-III (1971), avant d'être élu à l'EPHE, en janvier 1972, à la suite du décès brutal de Jacques Lépissier. La direction d'études « Histoire et philologie russe » qu'il prit alors devait durer près de trente ans, puisqu'il ne la quitta qu'avec son départ à la retraite, en juin 2001. Pendant ces trois décennies, il fut le plus souvent le seul universitaire français à dispenser un enseignement d'histoire médiévale russe. Toutefois, avec le perfectionnisme qui le caractérisait, il n'avait pas mis pour autant fin à ses études, puisqu'il soutint une thèse sur l'ensemble de ses travaux à l'université Paris-I - Panthéon-Sorbonne en 1985 : « Principauté russe et diplomatie princière $\left(\mathrm{X}^{\mathrm{e}}-\mathrm{XVI}^{\mathrm{e}} \mathrm{s}\right.$. $) »$.

Les années passées à l'EPHE permirent à Vladimir Vodoff de développer, devant un auditoire où historiens et linguistes se croisaient en bonne intelligence, une recherche embrassant le long Moyen Âge slave oriental, du X ${ }^{\mathrm{e}}$ au XVII ${ }^{\mathrm{e}}$, voire au XVIII ${ }^{\mathrm{e}}$ siècle. Plusieurs grands axes s'en dégagent. En premier lieu, un goût marqué, chartiste, pour les sciences auxiliaires de l'histoire et pour la bibliographie. Vladimir Vodoff circulait rarement sans son petit fichier de poche, qu'il enrichissait régulièrement de notations défiant parfois la clairvoyance de ses collègues paléographes. Avec patience et 
rigueur, il a composé et publié plusieurs mises au point sur la lexicographie du vieuxrusse, la diplomatique et les éditions de sources, les chroniques et l'historiographie. Il a dirigé la compilation du répertoire Histoire des Slaves orientaux des origines à 1689 : bibliographie des sources traduites en langues occidentales (1998). Il a aussi rédigé de très nombreux comptes rendus d'ouvrages dont la précision détaillée montrait l'attention avec laquelle ils avaient été lus et dans lesquels on peut puiser une mine de références utiles.

Son deuxième domaine de prédilection était l'histoire de l'Église. Il s'est exprimé à travers de copieux articles, toujours soigneusement référencés, dont les principaux ont été réunis en 2003 sous le titre Autour de la Sainte Russie. Il a trouvé son couronnement dans la rédaction du livre Naissance de la chrétienté russe, paru en 1988, année de la commémoration du millénaire du baptême de la Rus'. C'est à cette occasion que l'auteur a définitivement abandonné le « W $》$ un peu démodé que l'état-civil français avait conféré à l'initiale de son prénom, au profit du « V », plus directement en harmonie avec l'orthographe russe du prince Vladimir/Volodimer, auteur du baptême de 988.

Le troisième axe de réflexion fut l'histoire de la fonction princière, vue plus particulièrement sous l'angle de la terminologie. Les études de Vladimir Vodoff sur l'histoire du terme «tsar » dans les sources russes doivent ici être mentionnées comme un de ses apports les plus féconds. Ces textes et d'autres ont été rassemblés en 1989 dans un recueil intitulé Princes et principautés russes : $X^{e}-X V I I^{e} s$. Dans la mosaïque des principautés russes, il avait au fil du temps développé un intérêt particulier pour le cas particulier de Tver', rivale malheureuse de Moscou pour la suprématie dans les territoires du Nord-Est, à l'époque où s'esquisse le « rassemblement des terres russes » $\left(\mathrm{XIV}^{\mathrm{e}}-\mathrm{XV}^{\mathrm{e}} \mathrm{s}\right.$.).

Enfin, le dernier grand centre d'intérêt de Vladimir Vodoff a été Novgorod et la découverte, contemporaine de sa maturité scientifique, des écrits sur écorce de bouleau. Quoi de plus fascinant pour un paléographe, cotoyant des spécialistes du cunéiforme, des idéogrammes et des écritures alphabétiques les plus diverses, que de voir se constituer au fil des ans, dans son aire linguistique d'expertise, un corpus de documents médiévaux inconnus? Il faudrait imaginer une pierre de Rosette en miettes qui aurait été patiemment tirée d'un sol boueux, et non des sables d'Égypte, éclat par éclat, au cours d'une quarantaine d'années. C'est l'aventure que vivent encore nos collègues russes chaque été, depuis 1951. Vladimir Vodoff est celui qui a fait connaître au public français, à travers deux grands articles publiés dans le Journal des savants en 1966 et 1981, ces documents curieux et complexes, qui éclairent la vie quotidienne et ses tracas, souvent sans aucun apprêt stylistique, et nous semblent parfois si familiers.

Érudit exigeant, au point de paraître parfois austère, Vladimir Vodoff maniait aussi l'humour quand on le connaissait bien, savait faire preuve d'une grande disponibilité et de goût pour l'échange, avec les médiévistes comme avec les modernistes. Il aimait à dialoguer sur les sujets les plus divers : les fourrures et la météorologie de Novgorod avec Robert Delort, la christianisation avec Jean Delumeau, les imposteurs et autres « rois cachés » avec Yves-Marie Bercé. Les deux numéros thématiques de la Revue des études slaves qu'il a dirigés témoignent de cette ouverture d'esprit par leur thème et par la variété de leurs contributeurs. Le premier, s'intitulait Rus' de Kiev et Russie mos- 
covite : culture et société (t. 63.1, 1991). Le second, comparatiste, avait pour thème La Chrétienté latine et les Slaves orientaux (t. 70.2, 1998). La liste quasi complète des travaux de V. Vodoff est parue en 2003, au moment de la sortie d'un petit livre, Autour du Moyen Âge russe, qui rendait hommage à son enseignement en rassemblant l'intégralité des rapports qu'il avait rédigés pour le Livret-Annuaire de l'EPHE. Depuis, malheureusement, la maladie lui avait laissé fort peu de répit, l'empêchant de poursuivre ses travaux, comme il en avait l'intention, et de nous donner quelques-uns des inédits que ses notes conservent.

Nos pensées vont à sa femme, ses enfants et ses petits-enfants.

Pierre GonNEAU 